Pacific Journal of Mathematics

CLASSIFYING SPECIAL OPERATORS BY MEANS OF
SUBSETS ASSOCIATED WITH THE NUMERICAL RANGE 


\title{
CLASSIFYING SPECIAL OPERATORS BY MEANS OF SUBSETS ASSOCIATED WITH THE NUMERICAL RANGE
}

\author{
MARY R. EMBRY
}

Let $A$ be a continuous linear operator on a complex Hilbert space $X$, with inner product $<,>$ and associated norm \|\| . For each complex number $z$ let $M_{z}(A)=\{x:\langle A x, x\rangle=$ $\left.z\|x\|^{2}\right\}$. The following classifications of special operators are obtained: (i) $A$ is a scalar multiple of an isometry if and only if $A M_{z}(A) \subset M_{z}(A)$ for each complex $z$; (ii) $\mathbf{A}$ is a nonzero scalar multiple of a unitary operator if and only if $A M_{z}(A)=M_{z}(A)$ for each complex $z$; and (iii) $A$ is normal if and only if for each complex $z\left\{x \mid A x \in M_{z}(A)\right\}=\left\{x \mid A^{*} x \in M_{z}(A)\right\}$.

1. Introduction. The sets, $M_{z}(A)$, are closely associated with the numerical range of $A: W(A)=\{\langle A x, x\rangle:\|x\|=1\}$. These sets were introduced in [1] and used to characterize the elements of $W(A)$ as follows:

Theorem $A$. If $z \in W(A)$, then

(i ) $z$ is an extreme point of $W(A)$ if and only if $M_{z}(A)$ is linear,

(ii) if $z$ is a nonextreme boundary point of $W(A)$, then

$$
\gamma M_{z}(A)=\cup\left\{M_{w}(A): w \in L\right\}
$$

where $L$ is the line of support for $W(A)$ passing through $z$,

(iii) if $W(A)$ is a convex body, then $z$ is an interior point of $W(A)$ if and only if $\gamma M_{z}(A)=X$.

It was also shown in [1, Theorem 2] that $\cap$ \{maximal linear subspaces of $M_{z}(A)$ \} plays a special role in determining the normal eigenvalues of $A$.

With the aforementioned evidence concerning the sets $M_{z}(A)$ in mind, it seemed natural to ask whether these sets behave in a particular fashion if $A$ has special characteristics or whether the action of $A$ on these sets determines special properties of $A$. Obviously $A$ is Hermitian if and only if $M_{z}(A)=M_{z^{*}}(A)$ for all complex $z$. The first question which came to mind was: when is it the case that each of the sets $M_{z}(A)$ is invariant under $A$. The techniques developed to answer this question in Theorem 1 led to the other theorems in this paper. 
The following elementary facts can be noted about the sets, $M_{z}(A)$. 1. Each set $M_{z}(A)$ is homogeneous and 2. either $M_{z}(A) \cap M_{w}(A)=\{0\}$ or $M_{z}(A)=M_{w}(A)$.

2. Notation and terminology. The notation and terminology used in this paper are the same as that found in [1] with the following additions. $f$ is a bilinear functional on a complex vector space $X$ if and only if $f: X \times X \rightarrow$ complex numbers $\}, f$ is linear in the first variable and conjugate linear in the second variable.

Throughout the paper $A$ is a continuous linear operator on a complex Hilbert space $X ; A$ is an isometry if $A^{*} A=I ; A$ is unitary if $A^{*} A=A A^{*}=I ; A$ is normal if $A A^{*}=A^{*} A$; and $A$ is hyponormal if $A A^{*} \leqq A^{*} A$. ker $A$ denotes the null space of $A:\{x: A x=0\}$.

3. Classification theorems. The following lemma plays a fundamental part in the proofs of Theorems 1-4.

LEMmA 1. If $f, g, h$ and $k$ are bilinear functionals on a complex vector space $X$, satisfying

$$
\begin{aligned}
& f(x, x) g(x, x)=h(x, x) k(x, x) \text { for all } x \text { in } X \text {, then } \\
& f(x, y) g(x, y)=h(x, y) k(x, y) \text { for all } x \text { and } y \text { in } X .
\end{aligned}
$$

Indication of proof. Let $x, y \in X$ and let $z$ be an arbitrary complex number. By substituting $y+z x$ for $x$ in equation (1) and equating coefficients, one arrives at equation (2) by means of the coefficients of $z^{2}$.

THEOREM 1. $A$ is a scalar multiple of an isometry if and only if $A M_{z}(A) \subset M_{z}(A)$ for each complex $z$.

Proof. $M_{z}(A)$ is invariant under $A$ for each complex $z$ if and only if

$$
\left\langle A^{2} x, A x\right\rangle\|x\|^{2}=\langle A x, x\rangle\|A x\|^{2} \text { for all } x \text { in } X \text {. }
$$

Obviously if $A$ is a scalar multiple of an isometry, then equation (3) holds for all $x$ in $X$. Thus we assume that equation (3) holds for all $x$ in $X$ and by Lemma 1 have

$$
\left\langle A^{2} x, A y\right\rangle\langle x, y\rangle=\langle A x, y\rangle\langle A x, A y\rangle \text { for all } x \text { and } y \text { in } X .
$$

It now follows that $\{x\}^{\perp} \subset\{A x\}^{\perp} \cup\left\{A^{*} A x\right\}^{\perp}$. Moreover with $x$ and $y$ interchanged in (4) we see that $\{x\}^{\perp} \subset\left\{A^{*} x\right\}^{\perp} \cup\left\{A^{*} A x\right\}^{\perp}$. Since $\{y\}^{\perp}$ 
is linear, we have either $\{x\}^{\perp} \subset\left\{A^{*} A x\right\}^{\perp}$ or $\{x\}^{\perp} \subset\{A x\}^{\perp} \cap\left\{A^{*} x\right\}^{\perp}$. Either case implies that there exists a scalar $r_{x}$ such that $A^{*} A x=$ $\left(r_{x}\right) x$. This is sufficient to imply that $A$ is a scalar multiple of an isometry.

If $A$ is a nonunitary isometry, the only complex $z$ in $W(A)$ for which $A M_{z}(A)=M_{z}(A)$ are the extreme points of $W(A)$. To prove this we make use of results from [2] and [3] which assert that in this case $\sigma(A)=\overline{W(A)}=\{z:|z| \leqq 1\}$. Thus the elements of $W(A)$ are either extreme points $z$ with $|z|=1$ or interior points. If $z$ is an extreme point of $W(A)$, then since $A$ is hyponormal,

$$
M_{z}(A)=\left\{x: A x=z x \text { and } A^{*} x=z^{*} x\right\}
$$

by [4] and thus $M_{z}(A)=A M_{z}(A)=A^{*} M_{z}(A)$. Conversely if $M_{z}(A)=$ $A M_{z}(A)$, then $\gamma M_{z}(A)=A\left(\gamma M_{z}(A)\right)$. By Theorem A, (iii) if $z$ is an interior point of $W(A)$, then $X=A X$, implying that $A$ is invertible and hence unitary. Therefore if $M_{z}(A)=A M_{2}(A)$ and $z \in W(A)$, then $z$ is an extreme point of $W(A)$.

THEOREM 2. $A^{*}$ is a scalar multiple of an isometry if and only if $A^{*} M_{z}(A) \subset M_{z}(A)$ for each complex $z$.

Proof. Apply Theorem 1 to $A^{*}$ and note that $M_{z}\left(A^{*}\right)=M_{z^{*}}(A)$ for each complex $z$.

Theorem 3. $A$ is a nonzero scalar multiple of a unitary operator if and only if $A M_{z}(A)=M_{z}(A)$ for each complex $z$.

Proof. By Theorems 1 and $2 A$ is a scalar multiple of a unitary operator if and only if $A M_{z}(A) \subset M_{z}(A)$ and $A^{*} M_{z}(A) \subset M_{z}(A)$ for each complex $z$. Thus if $A$ is nonzero, this is equivalent to $A M_{z}(A) \subset M_{z}(A)$ and $M_{z}(A) \subset A M_{z}(A)$.

The proof of Theorem 4 which classifies normal operators in terms of the sets $M_{z}(A)$ appears to depend upon the following lemma.

Lemma 2. If $A$ and $E$ are operators on $X$ such that ker $A \subset k e r E$ and for each $x$ in $X$ either

or

(i ) $\|A x\|=\|E x\|$

(ii) there exists a real number $r_{x}$ such that

$$
A^{*} A x=\left(r_{x}\right) E^{*} E x,
$$

then $A^{*} A$ is a scalar multiple of $E^{*} E$. 
Proof. Assume that $A^{*} A x=a E^{*} E x$ and $A^{*} A y=b E^{*} E y$ where $E^{*} E x$ and $E^{*} E y$ are linearly independent. Let $t$ be real, $0<t<1$. Either $\| A(t x+(1-t) y\|=\| E(t x+(1-t) y \|$ or there exists a real number $c$ such that $A^{*} A(t x+(1-t) y)=c E^{*} E(t x+(1-t) y$. In this last case since $0<t<1$ and $E^{*} E x$ and $E^{*} E y$ are linearly independent, we have $a=c=b$. Thus if $a \neq b$, then

$$
\|A(t x+(1-t) y)\|=\|E(t x+(1-t) y)\|
$$

for all $t, 0<t<1$. Letting $t$ approach 1 and 0 , we have $\|A x\|=\|E x\|$ and $\|A y\|=\|E y\|$. Therefore $|a|=|b|=1$ and since $E^{*} E x \neq 0$ and $E^{*} E y \neq 0$, necessarily $a=b=1$. Thus we must have $a=b$ if $E^{*} E x$ and $E^{*} E y$ are linearly independent.

Secondly if $E^{*} E x$ and $E^{*} E y$ are linearly dependent and $A^{*} A x=$ $a E^{*} E x$ and $A^{*} A y=b E^{*} E y$, then it follows from the hypothesis $\operatorname{ker} A$ $\subset$ ker $E$ that $a$ and $b$ can be chosen to be the same real number.

The arguments in the two preceding paragraphs show that there exists a real number $r$ such that if $x \in X$, then either $A^{*} A x=r E^{*} E x$ or $\|A x\|=\|E x\|$. Thus either $\|A x\| \leqq\|E x\|$ for all $x$ in $X$ or $\|A x\| \geqq$ $\|E x\|$ for all $x$ in $X$. In either case $\{x:\|A x\|=\|E x\| E x \|\}$ is linear by Theorem $A$, (i). proving that $X$ is the union of the two linear subspaces:

$$
\left\{x: A^{*} A x=r E^{*} E x\right\} \quad \text { and } \quad\{x:\|A x\|=\|E x\|\} .
$$

Therefore either $A^{*} A=r E^{*} E$ or $A^{*} A=E^{*} E$.

THEOREM 4. $A$ is normal if and only if for each complex $z$

$$
\left\{x \mid A x \in M_{z}(A)\right\}=\left\{x \mid A^{*} x \in M_{z}(A)\right\} \text {. }
$$

Proof. If $A$ is normal it follows that $A x \in M_{z}(A)$ if and only if $A^{*} x \in M_{z}(A)$. Assume now that this condition holds. Then

$$
\left\langle A^{2} x, A x\right\rangle\left\|A^{*} x\right\|^{2}=\left\langle A A^{*} x, A^{*} x\right\rangle\|A x\|^{2} \text { for all } x \text { in } X
$$

and

$$
\operatorname{ker} A=\operatorname{ker} A^{*} \text {. }
$$

This last assertion can be proven as follows: $x \in \operatorname{ker} A \leftrightarrow A x \in M_{z}(A)$ for all complex $z \leftrightarrow A^{*} x \in M_{z}(A)$ for all complex $z \leftrightarrow x \in \operatorname{ker} A^{*}$.

Using the same techniques as in the proof of Theorem 1, we show that if $x \in X$, either their exists a number $b$ such that $A A^{*} x=b A^{*} A x$ or there exist numbers $c$ and $d$ such that $A A^{* 2} x=c A A^{*} x$ and $A^{*} A^{2} x=$ $d A^{*} A x$. These last two equations combined with (5) and (6) imply that either $A x=A^{*} x=0$ or $c=d^{*}$. They also imply that $A^{*^{2}} x=$ 
$c A^{*} x$ and $A^{2} x=d A x$. Again using (6), we have $A A^{*} x=c A x$ and $A^{*} A x=d A^{*} x$. Thus if $A x \neq 0,\left\|A^{*} x\right\|^{2}=c\langle A x, x\rangle=d^{*}\left\langle x, A^{*} x\right\rangle=$ $\|A x\|^{2}$. Therefore $A$ and $A^{*}$ satisfy the hypotheses of Lemma 2 and there exists a real number $r$ such that $A A^{*}=r A^{*} A$. This is sufficient to imply that $A$ is normal.

CoRollary 5. Let $A$ be an invertible operator on $X$. The following statements are equivalent:

(i) $A$ is normal,

(ii) $A^{-1} M_{z}(A)=A^{*-1} M_{z}(A)$ for each complex $z$,

(iii) $A^{-1} M_{z}\left(A^{*} A^{-1}\right)=A^{*-1} M_{z}\left(A^{*} A^{-1}\right)$ for each complex $z$.

Proof. The equivalence of (i) and (ii) is a restatement of Theorem 4 for the case in which $A$ is invertible. The equivalence of (i) and (iii) is obtained by applying Theorem 3 to the operator $A^{*} A^{-1}$.

I should like to express my appreciation to the referee of this paper for his helpful suggestions.

\section{REFERENCES}

1. M. R. Embry, The numerical range of an operator, Pacific J. Math., 32 (1970) 647-650.

2. C. R. Putnam, On semi-normal operators, Pacific J. Math., 7 (1957), 1649-1652.

3. - On the spectra of semi-normal operators, Trans. Amer. Math. Soc., 119 (1965), 509-523.

4. J. G. Stampfi, Extreme points of the numerical range of a hyponormal operator, Mich. Math. J., 13 (1966), 87-89.

Received June 16, 1970 and in revised form October 5, 1970.

University of North Carolina at Charlotte 



\section{PACIFIC JOURNAL OF MATHEMATICS}

\section{EDITORS}

H. SAMELSON

Stanford University

Stanford, California 94305

C. R. Hовву

University of Washington

Seattle, Washington 98105
J. DUGUNDJI

Department of Mathematics

University of Southern California

Los Angeles, California 90007

RICHARD ARENS

University of California

Los Angeles, California 90024

\section{ASSOCIATE EDITORS}
E. F. BECKENBACH
B. H. NeumanN
F. WOLF
K. YoSHIDA

\section{SUPPORTING INSTITUTIONS}

UNIVERSITY OF BRITISH COLUMBIA

CALIFORNIA INSTITUTE OF TECHNOLOGY

UNIVERSITY OF CALIFORNIA

MONTANA STATE UNIVERSITY

UNIVERSITY OF NEVADA

NEW MEXICO STATE UNIVERSITY

OREGON STATE UNIVERSITY

UNIVERSITY OF OREGON

OSAKA UNIVERSITY

UNIVERSITY OF SOUTHERN CALIFORNIA
STANFORD UNIVERSITY

UNIVERSITY OF TOKYO

UNIVERSITY OF UTAH

WASHINGTON STATE UNIVERSITY

UNIVERSITY OF WASHINGTON

AMERICAN MATHEMATICAL SOCIETY CHEVRON RESEARCH CORPORATION NAVAL WEAPONS CENTER 


\section{Pacific Journal of Mathematics}

\section{Vol. 38, No. $1 \quad$ March, 1971}

Bruce Alan Barnes, Banach algebras which are ideals in a Banach algebra ..... 1

David W. Boyd, Inequalities for positive integral operators............... 9

Lawrence Gerald Brown, Note on the open mapping theorem .............. 25

Stephen Daniel Comer, Representations by algebras of sections over Boolean

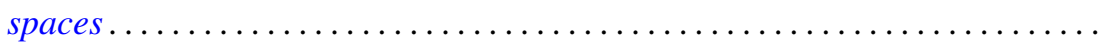

John R. Edwards and Stanley G. Wayment, On the nonequivalence of

conservative Hausdorff methods and Hausdorff moment sequences ........

P. D. T. A. Elliott, On the limiting distribution of additive functions $(\bmod 1) \ldots \ldots$

Mary Rodriguez Embry, Classifying special operators by means of subsets

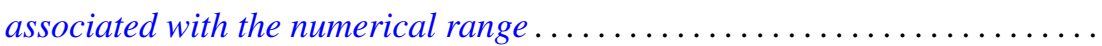

Darald Joe Hartfiel, Counterexamples to a conjecture of G. N. de Oliveira ......

C. Ward Henson, A family of countable homogeneous graphs...............

Satoru Igari and Shigehiko Kuratsubo, A sufficient condition for

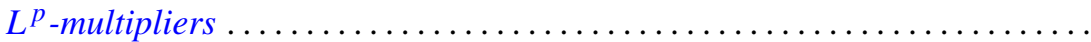

William A. Kirk, Fixed point theorems for nonlinear nonexpansive and

generalized contraction mappings............................

Erwin Kleinfeld, A generalization of commutative and associative rings ...... 95

D. B. Lahiri, Some restricted partition functions. Congruences modulo $11 \ldots \ldots 103$

T. Y. Lin, Homological algebra of stable homotopy ring $\pi *$ of spheres ....... 117

Morris Marden, A representation for the logarithmic derivative of a meromorphic function...........................

John Charles Nichols and James C. Smith, Examples concerning sum properties for metric-dependent dimension functions . .

Asit Baran Raha, On completely Hausdorff-completion of a completely

Hausdorff space.

M. Rajagopalan and Bertram Manuel Schreiber, Ergodic automorphisms and affine transformations of locally compact groups..........

N. V. Rao and Ashoke Kumar Roy, Linear isometries of some function

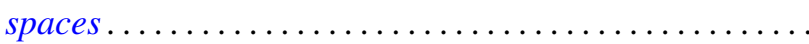

William Francis Reynolds, Blocks and F-class algebras of finite groups

Richard Rochberg, Which linear maps of the disk algebra are multiplicative ...

Gary Sampson, Sharp estimates of convolution transforms in terms of decreasing

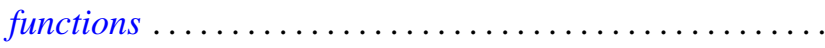

Stephen Scheinberg, Fatou's lemma in normed linear spaces

Ken Shaw, Whittaker constants for entire functions of several complex

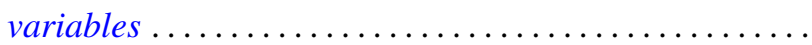

James DeWitt Stein, Two uniform boundedness theorems................ 251

$\mathrm{Li} \mathrm{Pi} \mathrm{Su,} \mathrm{Homomorphisms} \mathrm{of} \mathrm{near-rings} \mathrm{of} \mathrm{continuous} \mathrm{functions} \mathrm{.} \mathrm{.............} 261$

Stephen Willard, Functionally compact spaces, $C$-compact spaces and mappings of minimal Hausdorff spaces....................... 